\title{
Cannabis Extract Oil SR Capsule
}

National Cancer Institute

\section{Source}

National Cancer Institute. Cannabis Extract Oil SR Capsule. NCI Thesaurus. Code

C120551.

A sustained-release (SR), oil-based oral capsule composed of a cannabis extract, which contains a variety of cannabinoids but is comprised mainly of tetrahydrocannabinol (THC), with potential anti-cachexic and analgesic activities. Upon oral administration, the cannabinoids bind to the cannabinoid G-protein coupled receptor CB1, which is located in both central and peripheral neurons; CB1 receptor activation inhibits adenyl cyclase, increases various signal transduction pathways, and modulates the activity of various ion channels. This provides an analgesic effect, increases appetite, decreases chemotherapy-induced nausea and vomiting, and improves weight gain. The formulation allows for the immediate release of cannabinoids and quick onset of action, which is followed by a gradual release of cannabinoids. The SR form facilitates longer lasting therapeutic effects and causes fewer psychoactive side effects when compared to nonSR cannabinoid-containing oral formulations. 